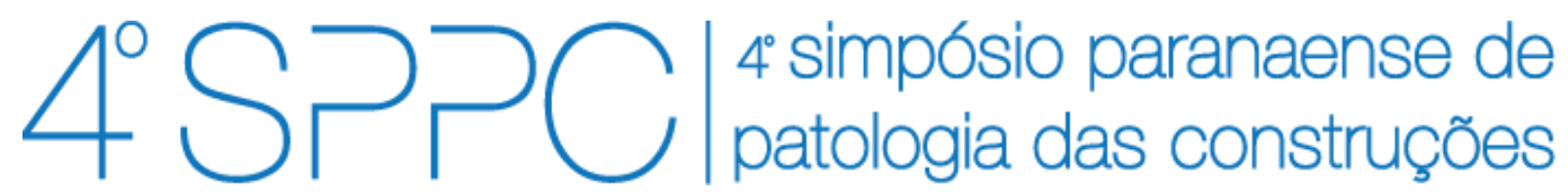

ISSN 2526-7248 artigo n. 4SPPC108, pp. 59-69, 2019

\title{
Correlação entre o ensaio de ultrassom e as propriedades mecânicas e de durabilidade de concretos convencionais
}

\author{
Amancio, Felipe Alves ${ }^{1}$; Dias, Alisson Rodrigues de Oliveira ${ }^{2 ;}$ Lucas, Sarah Oliveira ${ }^{3}$; \\ Lima, Douglas Alexandre ${ }^{4}$; Cabral, Antônio Eduardo Bezerra ${ }^{5}$; \\ 1,2 Mestre em Engenharia Civil, Universidade Federal do Ceará, \\ felipeaamancio@hotmail.com, rodrigues_alisson@live.com, \\ ${ }^{3,4}$ Graduando em Engenharia Civil, Universidade Federal do Ceará, \\ saraholiveiraluks@gmail.com, douglasalexandre@alu.ufc.br \\ ${ }^{5}$ Doutor em Engenharia Civil, Universidade Federal do Ceará, \\ eduardo.cabral@ufc.br
}

Resumo: Na construção civil, os ensaios não destrutivos (END) têm ganhado cada vez mais destaque, por serem um método de simples execução e baixo custo, na maioria dos casos. Com isto, o presente trabalho teve por objetivo investigar a correlação entre a velocidade de propagação ultrassônica (VPU) e as propriedades mecânicas e de durabilidade de concretos convencionais. Para isso, foram produzidos traços de concreto com classes de resistência de 20, 30 e $40 \mathrm{MPa}$ e ensaiados quanto à VPU, resistência à compressão, tração por compressão diametral, módulo de elasticidade estático, absorção de água por imersão, índice de vazios, capilaridade e penetração acelerada de íons cloreto. Pode-se constatar que existe uma boa correlação entre a VPU e as propriedades mecânicas e de durabilidade dos concretos, onde os concretos com maiores valores de VPU apresentaram menores valores de absorção de água, índice de vazios e penetração de cloretos, bem como melhor desempenho nas propriedades mecânicas.

Palavras-chave: Concreto, VPU, Ultrassom, Ensaios não destrutivos.

Abstract: In civil construction, the non-destructive tests (NDT) have gained increasing importance, since it is a method of simple execution and low cost, in most cases. The aim of the present work was to investigate the correlation between the ultrasonic propagation velocity (UPV) and the mechanical and durability properties of conventional concretes. For this, were produced mixes of concrete with resistance classes of 20,30 and $40 \mathrm{MPa}$ and tested for UPV, compressive strength, tensile strength by diametral compression, static modulus of elasticity, water absorption, void index, capillarity and accelerated chloride ion penetration. It can be verified that there is a good correlation between the UPV and the mechanical and durability properties of concretes, which the concretes with higher values of UPV presented lower values of water absorption, voids index and chloride penetration, as well as better mechanical properties.

Keywords: Concrete, UPV, Ultrasonic, Non-destructive tests. 
AMANCIO, F. A.; DIAS, A. R. O.; LUCAS, S. O.; LIMA, D. A.; CABRAL, A. E. B, CORRELAÇÃO ENTRE O ENSAIO DE ULTRASSOM E AS PROPRIEDADES MECÂNICAS E DE DURABILIDADE DE CONCRETOS CONVENCIONAIS. $4^{\circ}$ Simpósio Paranaense de Patologia das Construções (40 SPPC), artigo 4SPPC108, pp. 59 - 69, 2019. DOI: 10.4322/2526-7248.029

\section{Introdução}

Os Ensaios Não Destrutivos (END) são aqueles que não causam nenhum dano ao elemento ensaiado ou deixam pequenos danos para serem reparados após o ensaio, não provocando, assim, perda da capacidade resistente do elemento [1]. Tais ensaios permitem aos engenheiros avaliar e monitorar, de forma rápida e eficaz, estruturas de concretos. Estes métodos são usados para detectar os danos e para monitorar as estruturas na construção civil [2].

Os END são de extrema importância não somente para estruturas previamente executadas, mas também para estruturas novas ou ainda em execução, onde podem ser realizados ensaios de aceitação [3]. Dentre os END mundialmente utilizados, o método de determinação da Velocidade de Propagação Ultrassônica (VPU) foi desenvolvido tendo como objetivo principal a obtenção de curvas de correlação entre a VPU no concreto e a sua resistência à compressão [1].

A utilização de ensaios de velocidade de propagação do pulso ultra-sônico permite realizar um acompanhamento das características do material ao longo de sua vida útil. Através da análise dos dados obtidos, pode-se averiguar a uniformidade do concreto, controlar a sua qualidade e acompanhar sua deterioração, através de comparação com corpos de prova de referência [4]. Ademais, o uso destas técnicas permitem ainda investigar a massa específica, módulo de elasticidade e a resistência à compressão. Também podem ser investigadas a dureza superficial, a absorção, a permeabilidade, as condições de umidade, a localização das armaduras, a existência de vazios e a fissuração [3].

Neste sentido, o presente trabalho visa realizar uma correlação entre a VPU e as propriedades mecânicas e de durabilidade de concretos de cimento Portland produzidos com diferentes classes de resistência.

\section{Materiais e métodos}

\subsection{Materiais}

Nesta pesquisa foi utilizado cimento Portland CP V - ARI. As Tabelas 1 e 2 apresentam as características físicas e químicas do cimento fornecidas pelo fabricante.

Tabela 1: Resistência à compressão em MPa do cimento utilizado, por idade.

\begin{tabular}{ccc}
\hline Idade (dias) & Média (MPa) & $\begin{array}{c}\text { Especificação } \\
\text { Norma NBR 16697/2018 }\end{array}$ \\
\hline 1 & 25,9 & $\geq 14,0 \mathrm{Mpa}$ \\
3 & 36,6 & $\geq 24,0 \mathrm{Mpa}$ \\
7 & 44,3 & $\geq 34,0 \mathrm{Mpa}$ \\
28 & 53,2 & - \\
\hline
\end{tabular}


AMANCIO, F. A.; DIAS, A. R. O.; LUCAS, S. O.; LIMA, D. A.; CABRAL, A. E. B, CORRELAÇÃO ENTRE O ENSAIO DE ULTRASSOM E AS PROPRIEDADES MECÂNICAS E DE DURABILIDADE DE CONCRETOS CONVENCIONAIS. $4^{\circ}$ Simpósio Paranaense de Patologia das Construções (40 SPPC), artigo 4SPPC108, pp. 59 - 69, 2019. DOI: 10.4322/2526-7248.029

Tabela 2: Características físicas e químicas do cimento utilizado

\begin{tabular}{lccc}
\hline Ensaio & Norma & Resultado & $\begin{array}{c}\text { Especificação - Norma } \\
\text { NBR 16697/2018 }\end{array}$ \\
\hline Perda ao fogo & $\mathrm{NM} \mathrm{18/12}$ & $4,39 \%$ & $\leq 6,5$ \\
Óxido de Magnésio - MgO & $\mathrm{NM} \mathrm{11-2/12}$ & $4,17 \%$ & $\leq 6,5$ \\
Anidrido Sulfúrico - $\mathrm{SO}_{3}$ & $\mathrm{NM} \mathrm{16/12}$ & $3,24 \%$ & $\leq 4,5$ \\
Resíduo Insolúvel & $\mathrm{NM} \mathrm{15/12}$ & $0,69 \%$ & $\leq 3,5$ \\
Anidrido Carbônico - $\mathrm{CO}_{2}$ & $11578 / 91$ & $2,97 \%$ & $\leq 5,5$ \\
Massa específica & $\mathrm{NM} \mathrm{23/01}$ & $3,06 \mathrm{~g} / \mathrm{cm}^{3}$ & $\leq 6,0$ \\
Finura (\#325) & $12826 / 14$ & $4,80 \%$ & $\geq 1: 00 \mathrm{~h}: \mathrm{min}$ \\
Início de pega & $\mathrm{NM} \mathrm{65/03}$ & $2: 15 \mathrm{~h}: \mathrm{min}$ & $\leq 10: 00 \mathrm{~h}: \mathrm{min}$ \\
Fim de pega & $\mathrm{NM} \mathrm{65/03}$ & $3: 45 \mathrm{~h}: \mathrm{min}$ & $\leq 5: 00 \mathrm{~h}: \mathrm{min}$ \\
Expansibilidade a quente & $11582 / 16$ & $0,00 \mathrm{~mm}$ & $\leq 6,5$ \\
\hline
\end{tabular}

A água utilizada atende aos requisitos da ABNT NBR 15900-1:2009 [5]. O aditivo utilizado foi do tipo superplastificante da linha MasterGlenium ACE 402, composto por carboxilatos, à base de naftaleno sulfonato. $O$ aditivo possui possui $\mathrm{pH}$ de 7 , densidade de $1,15 \mathrm{~g} / \mathrm{cm}^{3}$ e solubilidade em água de $100 \mathrm{~g} / \mathrm{L}$. Os agregados miúdos e graúdos utilizados foram provenientes da Região Metropolitana de Fortaleza, atendendo aos requisitos da ABNT NBR 7211:2009 [6] A Tabela 03 apresenta as propriedades físicas dos agregados utilizados. A granulometria dos agregados miúdos é apresentada na Figura 1.

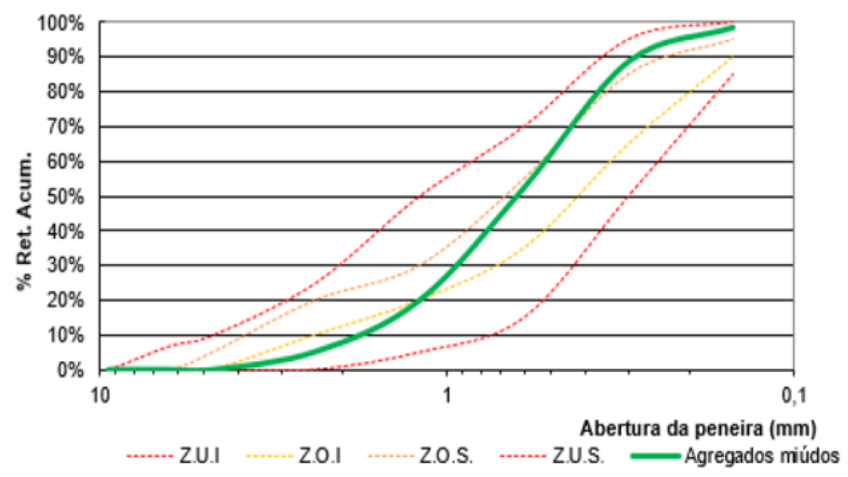

Figura 1: Curva granulométrica do agregado miúdo

Tabela 3: Propriedades físicas dos agregados utilizados

\begin{tabular}{ccccc}
\hline \multirow{2}{*}{ Ensaio } & \multirow{2}{*}{ Unidade } & \multicolumn{3}{c}{ Agregado } \\
\cline { 3 - 5 } & & Areia & Brita 12,5 $\mathbf{~ m m}$ & Brita 19mm \\
\hline Diâmetro máximo característico & $\mathrm{mm}$ & 4,75 & 12,5 & 19 \\
Módulo de finura & Adim & 2,65 & 5,99 & 6,84 \\
Massa unitária & $\mathrm{g} / \mathrm{cm}^{3}$ & 1,41 & 1,41 & 1,37 \\
Absorção de água & $\%$ & 0,9 & 0,89 & 0,94 \\
Massa específica & $\mathrm{g} / \mathrm{cm}^{3}$ & 2,59 & 2,61 & 2,61 \\
Material pulverulento & $\%$ & 1,0 & 0,29 & 0,9 \\
\hline
\end{tabular}


AMANCIO, F. A.; DIAS, A. R. O.; LUCAS, S. O.; LIMA, D. A.; CABRAL, A. E. B, CORRELAÇÃO ENTRE O ENSAIO DE ULTRASSOM E AS PROPRIEDADES MECÂNICAS E DE DURABILIDADE DE CONCRETOS CONVENCIONAIS. $4^{\circ}$ Simpósio Paranaense de Patologia das Construções (40 SPPC), artigo 4SPPC108, pp. 59 - 69, 2019. DOI: 10.4322/2526-7248.029

\subsection{Métodos de pesquisa}

Os traços adotados foram provenientes de central dosadora da cidade de Fortaleza, Ceará, por serem os mais comercializados na região. Os traços possuem classes de resistência C20, C30 e C40, devendo atingir aos 28 dias as resistências de $20 \mathrm{MPa}$, $30 \mathrm{MPa}$ e $40 \mathrm{MPa}$, respectivamente. A consistência no estado fresco foi fixada em $100 \pm 10$ milímetros de abatimento do tronco de cone realizado por meio do ensaio slump test, conforme ABNT NBR NM 67:1998 [7], sendo este corrigido por meio do aumento do teor de aditivo, não sendo alterado o fator a/c dos traços. A Tabela 4 apresenta o consumo de materiais dos traços investigados, em kg/ $\mathrm{m}^{3}$.

Tabela 4: Consumo de materiais em $\mathrm{kg} / \mathrm{m}^{3}$ para os traços investigados.

\begin{tabular}{ccccccc}
\hline Classe & Cimento & Areia & $\begin{array}{c}\text { Brita } \\
\mathbf{1 2 , 5 m m}\end{array}$ & $\begin{array}{c}\text { Brita } \\
\mathbf{1 9} \mathbf{~ m m}\end{array}$ & Aditivo (\%) & a/c \\
\hline C20 & 260 & 860 & 277 & 694 & 0,37 & 0,69 \\
C30 & 360 & 794 & 264 & 703 & 0,28 & 0,50 \\
C40 & 440 & 710 & 266 & 709 & 0,33 & 0,41 \\
\hline
\end{tabular}

No estado fresco, após a determinação da trabalhabilidade por meio do ensaio slump test, deu-se início a moldagem dos corpos de prova cilíndricos de $10 \mathrm{~cm}$ de diâmetro e $20 \mathrm{~cm}$ de altura e cura dos mesmos, realizada por imersão em tanques de água saturada com cal, expostos à temperatura ambiente até a data de ensaio. Em seguida, realizou-se os ensaios de caracterização dos concretos no estado endurecido aos 28 dias, tendo sido utilizado 3 corpos de prova para cada ensaio.

A resistência à compressão foi realizada conforme preconiza a ABNT NBR 5739:2018 [8]. O ensaio de resistência à tração por compressão diametral foi realizado conforme regulamenta a ABNT NBR 7222:2010 [9]. O módulo de elasticidade estático foi analisado para a idade de 28 dias, realizado conforme os procedimento da ABNT NBR 8522:2017 [10]. Em relação à durabilidade, foram realizados os seguintes ensaios: absorção de água por imersão e índice de vazios (ABNT NBR 9778:2009) [11], coeficiente de capilaridade (ABNT NBR 9779:2009) [12] e determinação da capacidade de penetração acelerada de íons, conduzida de acordo com a ASTM C 1202:2012 - Electrical Indication of Concrete's Ability to Resist Chloride Ion Penetration [13]. O método consiste em acoplar uma fatia de concreto de dimensões $10 \times 5 \mathrm{~cm}$ (diâmetro e altura) entre duas meia-células acrílicas, sendo uma preenchida com uma solução aquosa de cloreto de sódio ( $\mathrm{NaCl})$ com 3\% de concentração e outra com solução aquosa de hidróxido de sódio $(\mathrm{NaOH})$ com concentração de $0,3 \mathrm{~N}$. A avaliação da penetração de íons cloretos é feita mediante a medição da carga passante na amostra de concreto, submetida a uma tensão de $60 \pm 0,1 \mathrm{~V}$ durante um período de 6 horas. Entre as meias-células é gerada uma corrente elétrica que induz o ânion cloreto a se difundir através do concreto sob a ação de um campo elétrico. A leitura da corrente passante é registrada por um amperímetro, em intervalos de trinta minutos. A representação esquemática desse ensaio pode ser visto na Figura 2. 


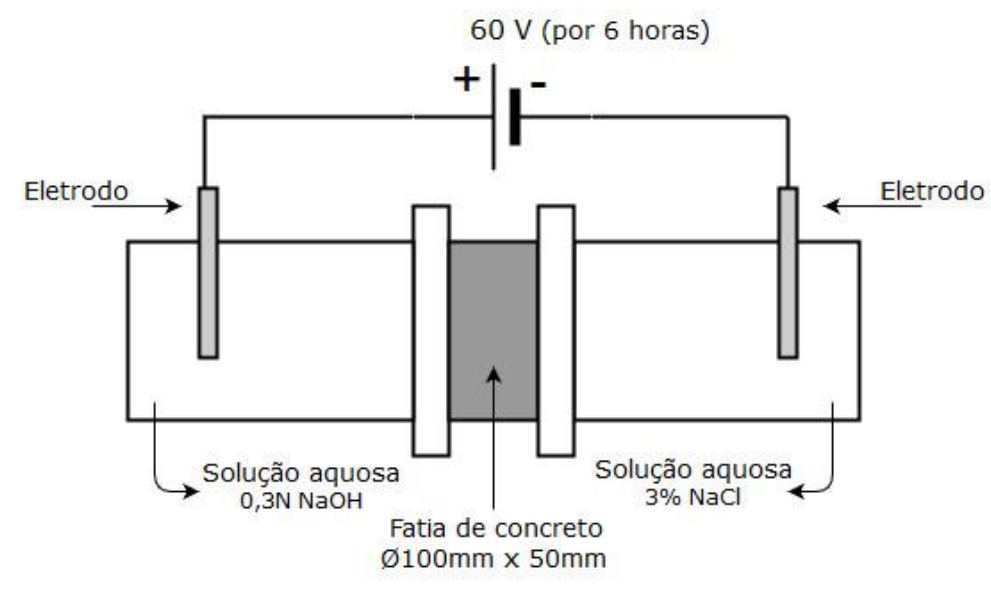

Figura 2: Representação esquemática do ensaio de penetração de íons cloretos.

Para o ensaio de penetração de íons cloretos os corpos de prova foram serrados em quatro partes perpendiculares ao eixo com dimensões de $10 \times 5 \mathrm{~cm}$ (diâmetro e altura) cada. É importante salientar que foram utilizadas as partes centrais dos corpos de prova, sendo descartadas as partes superiores e inferiores, de modo a desconsiderar o efeito de qualquer segregação e/ou exsudação que pudesse distorcer os resultados, para isso foram avaliados 4 amostras por traço.

A carga total passante foi obtida por meio da Equação 1, sendo $Q$ - carga total passante (coulombs, C); 10 - corrente (amperes) medida imediatamente após a aplicação da tensão; e It - corrente (amperes) medida nos intervalos "t" em minutos. A partir do resultado obtido, foi possível classificar qualitativamente o concreto quanto ao risco de penetração de íons cloretos, conforme a Tabela 5.

$$
Q=900 \cdot\left(I_{0}+2 \cdot I_{30}+2 \cdot I_{60}+\cdots+2 \cdot I_{330}+I_{360}\right) \text { Equação } 01
$$

Tabela 5: Classificação da penetração de íons cloretos no concreto

\begin{tabular}{cc}
\hline Carga passante $(\mathbf{C})$ & Risco de penetração de íons cloretos \\
\hline$>4000$ & Alto \\
2000 a 4000 & Moderado \\
1000 a 2000 & Baixo \\
100 a 1000 & Muito baixo \\
$<100$ & Desprezível \\
\hline
\end{tabular}

Fonte: ASTM C1202 [13].

Destaca-se que para o ensaio de penetração de íons cloretos, foram utilizados três amostras, enquanto para os demais ensaios de durabilidade foram utilizados apenas duas amostras. A determinação da velocidade de propagação ultrassônica 
(VPU) nos concretos foi realizada aos 28 dias, utilizando 3 corpos de prova, conforme os procedimentos estabelecidos na ABNT NBR 8802:2017 [14]. O equipamento utilizado foi um instrumento da Pundit Labda PROCEQ, com frequência dos transdutores na ordem de $54 \mathrm{~Hz}$.

\section{Resultados e Discussões}

A Tabela 6 apresenta os resultados gerais e médios obtidos para as propriedades dos concretos no estado endurecido. Com os valores contidos na Tabela 6 foi possível estabelecer as correlação entre a VPU e as propriedades mecânicas e durabilidade dos concretos.

Tabela 6: Resultados gerais e médios obtidos para as propriedades dos traços de concreto no estado endurecido.

\begin{tabular}{cccccccccc}
\hline & & \multicolumn{7}{c}{ Propriedades analisadas } \\
\cline { 3 - 10 } Classe & CP & VPU & $\begin{array}{c}\text { Resist. } \\
\text { comp. } \\
\text { (MPa) }\end{array}$ & $\begin{array}{c}\text { Tração } \\
\text { Diametral } \\
\text { (MPa) }\end{array}$ & $\begin{array}{c}\text { Módulo } \\
\text { elastic. } \\
\text { (GPa) }\end{array}$ & $\begin{array}{c}\text { Capil. } \\
\left(\mathbf{g} / \mathbf{c m}^{2}\right)\end{array}$ & $\begin{array}{c}\text { Carga } \\
\text { passante } \\
(\mathbf{C})\end{array}$ & $\begin{array}{c}\text { Ind. } \\
\text { vazios } \\
(\%)\end{array}$ & $\begin{array}{c}\text { Absorção } \\
\text { imersão } \\
(\%)\end{array}$ \\
\hline \multirow{4}{*}{ C20 } & CP 1 & 4343 & 22,69 & 1,99 & 24,79 & 0,267 & 4111 & 7,68 & 3,56 \\
& CP 2 & 4205 & 20,01 & 1,75 & 22,79 & 0,28 & 4183 & 7,18 & 3,25 \\
& CP 3 & 4159 & 19,92 & 1,74 & 19,32 & - & 4298 & - & - \\
& Média & 4236 & 20,87 & 1,83 & 22,3 & 0,274 & 4197 & 7,43 & 3,41 \\
\hline \multirow{4}{*}{ C30 } & CP 1 & 4548 & 30,79 & 2,55 & 23,18 & 0,204 & 3734 & 5,28 & 2,34 \\
& CP 2 & 4548 & 32,6 & 2,88 & 24,35 & 0,153 & 3668 & 6,56 & 3 \\
& CP 3 & 4717 & 33,12 & 3,13 & 27,51 & - & 3485 & - & - \\
& Média & 4604 & 32,17 & 2,85 & 25,01 & 0,179 & 3629 & 5,92 & 2,67 \\
\hline \multirow{6}{*}{ C40 } & CP 1 & 5339 & 42,38 & 3,00 & 31,77 & 0,115 & 1879 & 3,81 & 1,68 \\
& CP 2 & 5013 & 40,9 & 2,95 & 31,49 & 0,127 & 1970 & 3,87 & 1,71 \\
& CP 3 & 5013 & 40,93 & 2,9 & 29,16 & - & 1947 & - & - \\
& Média & 5122 & 41,40 & 2,95 & 30,81 & 0,121 & 1932 & 3,84 & 1,70 \\
\hline
\end{tabular}

As Figuras 3 e 4 apresentam a correlação da VPU com a resistência à compressão e a tração por compressão diametral, respectivamente. Constata-se que concretos com maiores valores de VPU apresentaram maiores valores de resistência mecânica. Isto pode ser justificado pelo fato desses concretos terem apresentado um maior consumo de cimento e um menor fator $\mathrm{a} / \mathrm{c}$, o que os tornam menos poroso, e consequentemente mais resistentes. 


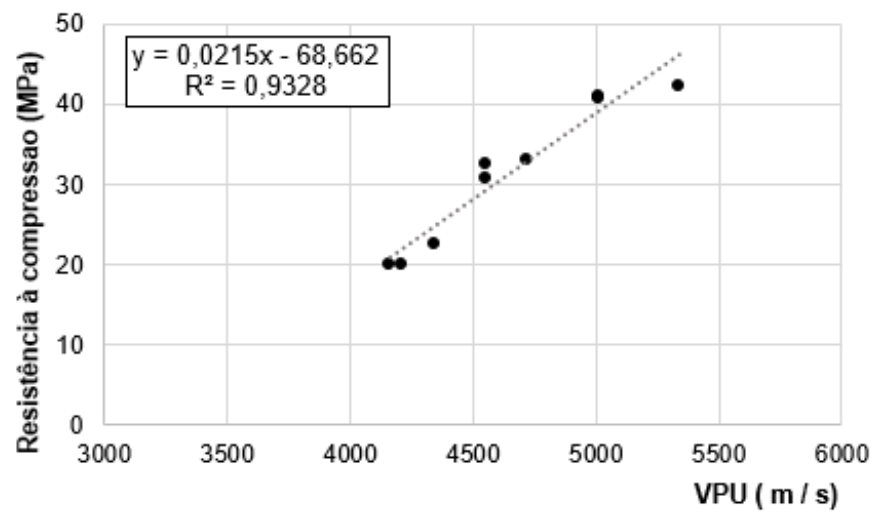

Figura 3: Correlação entre VPU e resistência à compressão.

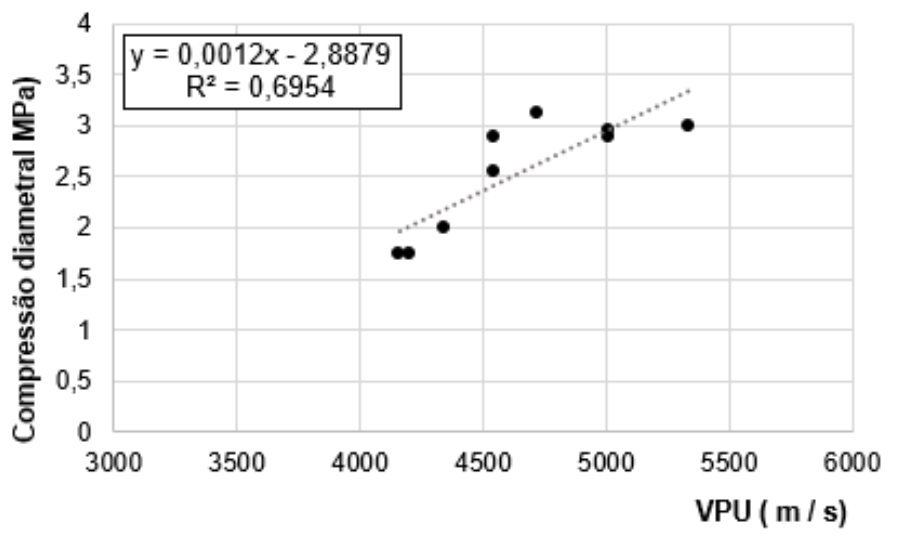

Figura 4: Correlação entre VPU e tração por compressão diametral.

Foi possível estabelecer uma ótima correlação entre a VPU e a resistência à compressão (Figura 3), com um valor de $\mathrm{R}^{2}$ de 0,93 , enquanto que a correlação entre a VPU e a tração por compressão diametral foi determinado um $\mathrm{R}^{2}$ na ordem de 0,69 (Figura 4).

Quanto aos valores de módulo de elasticidade estáticos obtido nos ensaios com extensômetros, foi possível estabelecer uma correlação com a VPU dos concretos (Figura 5). Concretos com maiores VPU apresentam maiores valores de módulo de elasticidade. Ressalta-se ainda que o nível de correlação foi elevado, tendo-se um $\mathrm{R}^{2}$ de 0,88 .

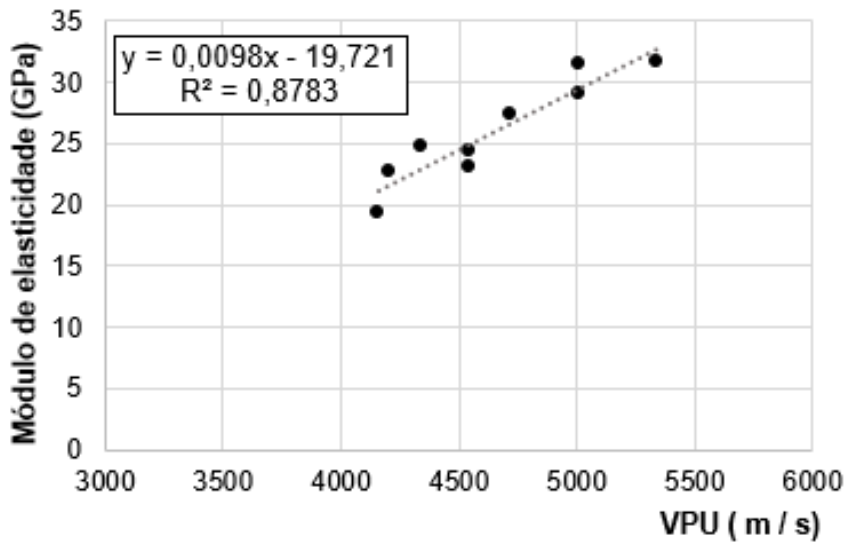

Figura 5 - Correlação entre VPU e módulo de elasticidade estático. 
As Figuras 6 a 8 apresentam a correlação da VPU com o índice de vazios, capilaridade e absorção de água por imersão, respectivamente. Destaca-se que os concretos com menores valores de VPU, os quais possuem menores consumo de cimento e maiores relação a/c foram os que apresentaram maiores valores nestas propriedades, o que os tornam de menor durabilidade. Devido à maior relação a/c nos concretos de menores resistências, estes apresentam uma maior porosidade, 0 que reflete nos menores valores de VPU e consequentemente nos resultados dos ensaios de durabilidade. Schulze [15] complementa ainda explicando que a absorção de água é reduzida com uma menor relação água-cimento. Com um teor crescente de cimento e uma relação água-cimento constante, a absorção de água aumenta.

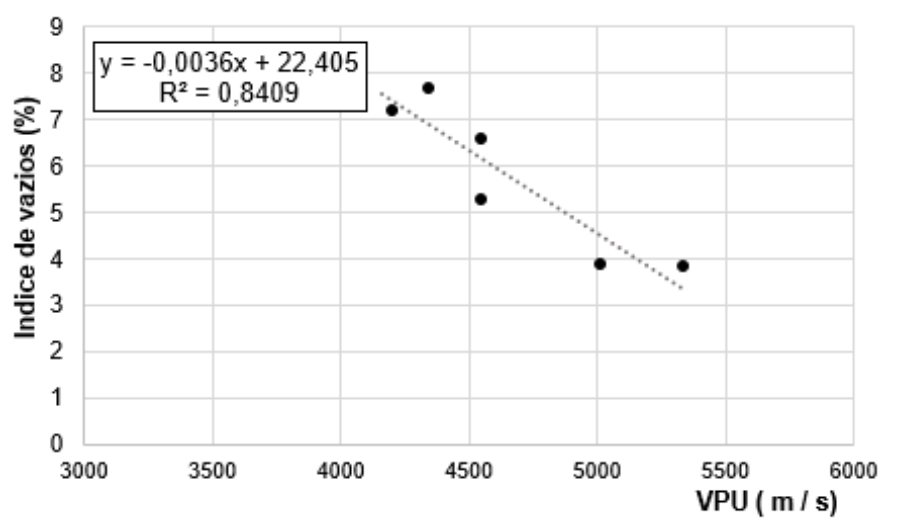

Figura 6 - Correlação entre VPU e índice de vazios.

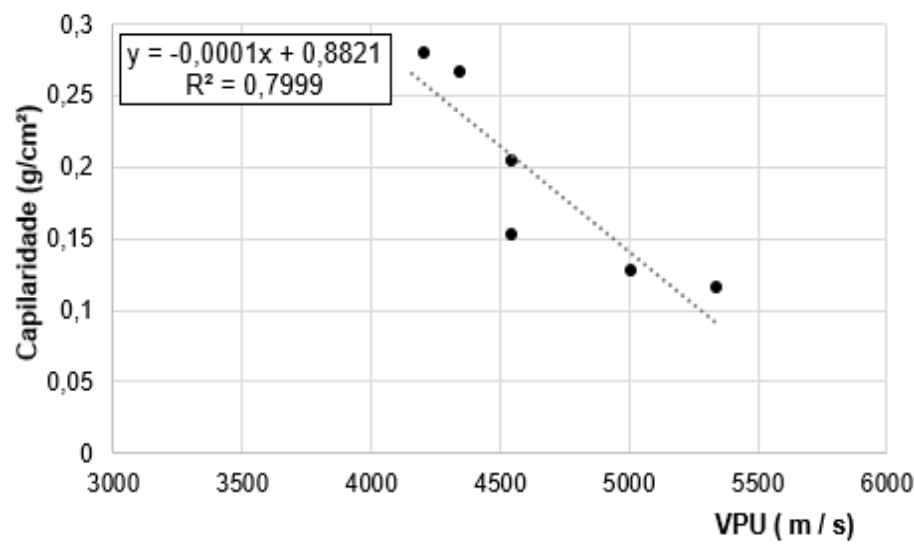

Figura 7: Correlação entre VPU e capilaridade.

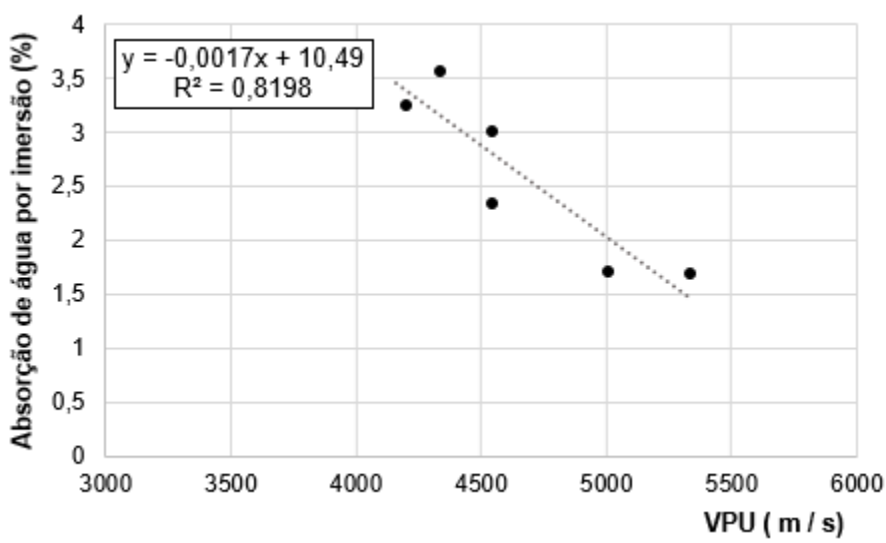

Figura 8: Correlação entre VPU e absorção de água por imersão. 
A Figura 9 apresenta a correlação entre a carga passante $(C)$ - obtida no ensaio de penetração de íons cloretos - e a VPU nos concretos. É possível observar que os concretos de maiores velocidades ultrassônicas, apresentaram menores valores de carga passante, o que os tornam mais resistentes a penetrabilidade de íons cloretos. Isso se deve ao fato de que esses concretos apresentam menor fator a/c, contribuindo para a redução da porosidade no estado endurecido, dificultando a penetração dos íons cloretos, e consequentemente, aumentando a durabilidade.

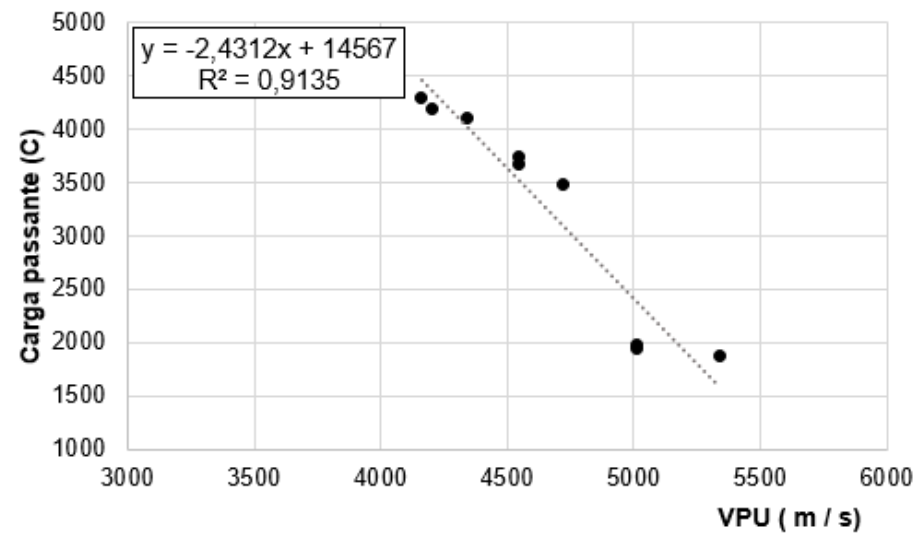

Figura 9: Correlação entre VPU e carga passante de íons cloreto.

Por fim, é apresentado na Tabela 7 um resumo das equações e os valores dos $\mathrm{R}^{2}$ das correlações entre a VPU e as propriedades mecânicas e de durabilidade do concreto.

Tabela 7: Resumo das correlações entre a VPU e as propriedades do concreto.

\begin{tabular}{cccc}
\hline Propriedade & Unid. & Equação & $\mathbf{R}^{\mathbf{2}}$ \\
\hline Resistência à compressão & $\mathrm{MPa}$ & $\mathrm{y}=0,0215 \mathrm{x}-68,662$ & 0,9328 \\
Tração por compressão diametral & $\mathrm{MPa}$ & $\mathrm{y}=0,0012 \mathrm{x}-2,8879$ & 0,6954 \\
Módulo de elasticidade & $\mathrm{Gpa}$ & $\mathrm{y}=0,0098 \mathrm{x}-19,721$ & 0,8783 \\
Índice de vazios & $\%$ & $\mathrm{y}=-0,0036 \mathrm{x}+22,405$ & 0,8409 \\
Capilaridade & $\mathrm{g} / \mathrm{cm}^{2}$ & $\mathrm{y}=-0,0001 \mathrm{x}+0,8821$ & 0,7999 \\
Absorção de água por imersão & $\%$ & $\mathrm{y}=-0,0017 \mathrm{x}+10,49$ & 0,8198 \\
Carga passante de íons cloretos & $\mathrm{C}$ & $\mathrm{y}=-2,4312 \mathrm{x}+14567$ & 0,9135 \\
\hline
\end{tabular}

\section{Considerações Finais}

O presente estudo teve por objetivo investigar as correlações entre as propriedades mecânicas e de durabilidade com a velocidade de propagação de ondas ultrassônicas (VPU) nos concretos convencionais. Constatou-se que as correlações entre VPU e resistência à compressão, bem como para módulo de elasticidade, apresentaram valores de $R^{2}$ bastante representativo, acima de 0,85 , diferentemente da correlação com a tração por compressão diametral que apresentou $R^{2}$ de 0,69.

Em relação às propriedades de durabilidade, foi possível estabelecer correlações com $\mathrm{R}^{2}$ representativos, com destaque para o ensaio de penetração acelerada de 
AMANCIO, F. A.; DIAS, A. R. O.; LUCAS, S. O.; LIMA, D. A.; CABRAL, A. E. B, CORRELAÇÃO ENTRE O ENSAIO DE ULTRASSOM E AS PROPRIEDADES MECÂNICAS E DE DURABILIDADE DE CONCRETOS CONVENCIONAIS. $4^{\circ}$ Simpósio Paranaense de Patologia das Construções (40 SPPC), artigo 4SPPC108, pp. 59 - 69, 2019. DOI: 10.4322/2526-7248.029

íons cloretos, o qual a correlação apresentou um $\mathrm{R}^{2}$ de 0,91 . Isso demonstra que existe uma viabilidade de se estimar a penetração de íons cloretos por meio da VPU do concreto.

Por fim, por meio das correlações estabelecidas, demonstra-se que a técnica de ultrassom apresenta viabilidade no intuito de monitorar e avaliar as propriedades mecânicas e durabilidade dos concretos por meio da VPU. Ademais, esta técnica se torna ainda mais atrativa em virtude da simples execução, bem como o baixo custo, torna-se viável a sua utilização.

\section{Agradecimentos}

Os autores agradecem ao Programa de Pós-Graduação em Engenharia Civil: Estruturas e Construção Civil (PEC) e ao Laboratório de Materiais de Construção Civil (LMCC) da Universidade Federal do Ceará - UFC. O presente trabalho foi realizado com apoio da Coordenação de Aperfeiçoamento de Pessoal de Nível Superior - Brasil (CAPES) - Código de Financiamento 001.

\section{Referências}

[1] ADAMATI, D. S.; LORENZI, A.; CHIES, J. A.; SILVA FILHO, L. C. P. Analysis of reinforced concrete structures through the ultrasonic pulse velocity: technological parameters involved IBRACON Structures and Materials Journal. vol. 10 n 2, 2017.

[2] REHMAN, S. K. R.; IBRAHIM, Z.; MEMON, S. A.; JAMEEL, M.; Nondestructive test methods for concrete bridges: A review. Construction and building materials. v.107, pp 58-86 março, 2016.

[3] EVANGELISTA, A. C. J., Avaliação da Resistência do Concreto usando Diferentes Ensaios Não Destrutivos. 2002. 219p. Tese (Doutorado) - Programa de PósGraduação em Engenharia da Universidade Federal do Rio de Janeiro. Universidade Federal do Rio de Janeiro, Rio de Janeiro, 2002.

[4] LORENZI, A. Desenvolvimento de Redes Neurais Artificiais para Avaliação de Estruturas de Concreto através de Ensaios não Destrutivos. 2009. Tese (Doutorado em Engenharia Civil). Programa de Pós-Graduação em Engenharia Civil, UFRGS, Porto Alegre, RS.

[5] ASSOCIAÇÃO BRASILEIRA DE NORMAS TÉCNICAS. NBR 15900-1. Água para amassamento do concreto. Requisitos. Rio de Janeiro, 2009.

[6] ASSOCIAÇÃO BRASILEIRA DE NORMAS TÉCNICAS. NBR 7211. Agregados para concreto. Especificação. Rio de Janeiro, 2009.

[7] ASSOCIAÇÃO BRASILEIRA DE NORMAS TÉCNICAS. NBR NM 67. Determinação da Consistência pelo abatimento do tronco de cone. Rio de Janeiro, 1998.

[8] ASSOCIAÇÃO BRASILEIRA DE NORMAS TÉCNICAS. NBR 5739. Concreto Ensaio de compressão de corpos de prova cilíndricos. Rio de Janeiro, 2018.

[9] ASSOCIAÇÃO BRASILEIRA DE NORMAS TÉCNICAS. NBR 7222. Concreto e argamassa- Determinação da resistência à tração por compressão diametral de corpos de prova cilíndricos. Rio de Janeiro, 2011. 
AMANCIO, F. A.; DIAS, A. R. O.; LUCAS, S. O.; LIMA, D. A.; CABRAL, A. E. B, CORRELAÇÃO ENTRE O ENSAIO DE ULTRASSOM

E AS PROPRIEDADES MECÂNICAS E DE DURABILIDADE DE CONCRETOS CONVENCIONAIS. $4^{\circ}$ Simpósio Paranaense de Patologia das Construções (40 SPPC), artigo 4SPPC108, pp. 59 - 69, 2019. DOI: 10.4322/2526-7248.029

[10] ASSOCIAÇÃO BRASILEIRA DE NORMAS TÉCNICAS. NBR 8522. Concreto Determinação dos módulos estáticos de elasticidade e de deformação à compressão. Rio de Janeiro, 2017.

[11] ASSOCIAÇÃO BRASILEIRA DE NORMAS TÉCNICAS. NBR 9778. Argamassa e concreto endurecidos - Determinação da absorção de água, índice de vazios e massa específica. Rio de Janeiro, 2005.

[12] ASSOCIAÇÃO BRASILEIRA DE NORMAS TÉCNICAS. NBR 9779. Argamassa e concreto endurecidos. Determinação da absorção de água por capilaridade. Rio de Janeiro, 2012.

[13] AMERICAN SOCIETY FOR TESTING AND MATERIALS. ASTM C 1202. Standard test method for electrical indication of concrete's ability to resist chloride ion penetration. Philadelphia, 2012.

[14] ASSOCIAÇÃO BRASILEIRA DE NORMAS TÉCNICAS. NBR 8802Concreto endurecido - Determinação da velocidade de propagação de onda ultrassônica. Rio de Janeiro, 2019.

[15] SCHULZE, J. Influence of water-cement ratio and cement content on the properties of polymer-modified mortars. Cement and Concrete Research. v. 29, pp.909-915, 1999. 